\title{
Anomalous thickness evolution of multilayer films made from poly-L-lysine and mixtures of hyaluronic acid and polystyrene sulfonate
}

Grégory Francius ${ }^{1,2}$, Joseph Hemmerlé ${ }^{1,2}$, Jean-Claude Voegel ${ }^{1,2}$, Pierre Schaaf ${ }^{3}$, Bernard Senger ${ }^{1,2}$ and Vincent Ball ${ }^{1,2}$

1. Institut National de la Santé et de la Recherche Médicale, INSERM Unité 595, 11 rue Humann, 67085 Strasbourg Cedex, France

2. Faculté de Chirurgie Dentaire, Université Louis Pasteur, 1 place de l'Hôpital, 67000 Strasbourg, France

3. Centre National de la Recherche Scientifique, Unité Propre de Recherche 22, Institut Charles Sadron, 6 rue Boussingault, 67083 Strasbourg Cedex, France

Corresponding author: vincent.ball@medecine.u-strasbg.fr

\section{Supporting information}

Figure 5 shows the evolution of the HA mass fraction, $y$, in a film constituted of 12 pairs of layers built on top of the PEI precursor layer, as a function of the HA mass fraction in the buildup solution, $x$. The corresponding PSS mass fraction in the same film is $1-y$. The striking feature of Figure 2 is the non-monotonous evolution of the film thickness when $x$ ranges from 0 up to 1 , although $y$ increases monotonically with $x$ and, consequently, $1-y$ decreases monotonically with $x$. It is the aim of this supporting information section to show that these observations are compatible.

The evolution of $y$ with $x$ can be represented empirically by:

$$
y=a x+(1-a) x^{\mathrm{p}}
$$

where $a$ and $p$ are free parameters. The mass fraction can be converted into the molar fraction, $z$, of HA in the same film: 


$$
z=\frac{M_{\mathrm{PSS}} y}{M_{\mathrm{HA}}(1-y)+M_{\mathrm{PSS}} y},
$$

with $M_{\mathrm{HA}}=400 \mathrm{~kg} / \mathrm{mol}$ and $M_{\mathrm{PSS}}=70 \mathrm{~kg} / \mathrm{mol}$. The corresponding mass fraction and molar fraction of PSS are $1-y$ and $1-z$, respectively. Both $y$ and $z$ are represented in Figure A1, with $a=0.25$ and $p=15$.

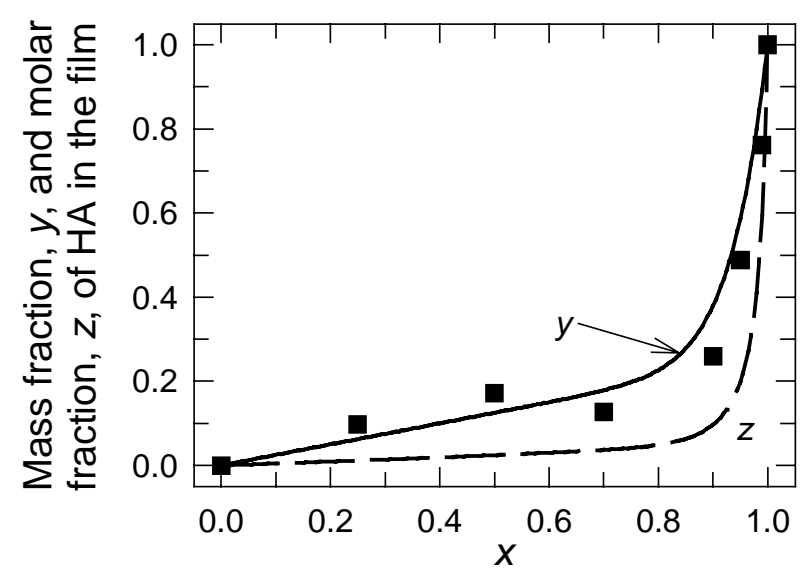

Figure A1. Mass fraction of HA in the film PEI-[(HA $\left.\left.-\mathrm{PSS}_{1-\mathrm{x}}\right)-\mathrm{PLL}\right]_{12}$ as a function of the HA mass fraction, $x$, in the polyanion buildup solution. The squares represent $y$ deduced from the infrared spectroscopy measurements and the solid line, labeled " $y$ ", corresponds to Eq. (A1) with $a=0.25$ and $p=15$. The dashed line represents the molar fraction $z$ (Eq. (A2)).

Figure A2 represents the thickness of PEI-[(HA $\left.\left.-\mathrm{HSS}_{1-\mathrm{x}}\right)-\mathrm{PLL}\right]_{\mathrm{n}}$ films measured by ellipsometry, as a function of the HA content, $x$, of the blended polyanion solution, for $n=14$ (a), 12 (b), 10 (c) and 8 (d). The data points corresponding to $n=14$ were already given in Figure 2 with other measurements that have been withdrawn here for the sake of clarity.

From here on, we assume that $y$ is invariant with respect to $n$ at a fixed value of $x$. If we draw $\beta(1-y)$, where $\beta$ means the thickness when the polyanion solution contains only PSS $(x=0)$, as a function of the composition variable $x$, we observe that this function reproduces well the measured film thickness over a wide domain of $x$ extending up to about 0.90 (Figure A2). The values of $\beta$ are given in Table 1. This suggests two conclusions, 
namely (i) that the film thickness is mainly (if not entirely) due to the interaction of PSS with PLL when $x \leq 0.90$ and (ii) that the thickness is proportional to the mass fraction rather than to the molar fraction of PSS. It is here worth reminding that films built up with the polyanion solution containing exclusively PSS had the same thickness as those built with the blended polyanion solution when the PSS concentration was the same $(n=14$, Figure 2$)$. This reinforces conclusion (i).
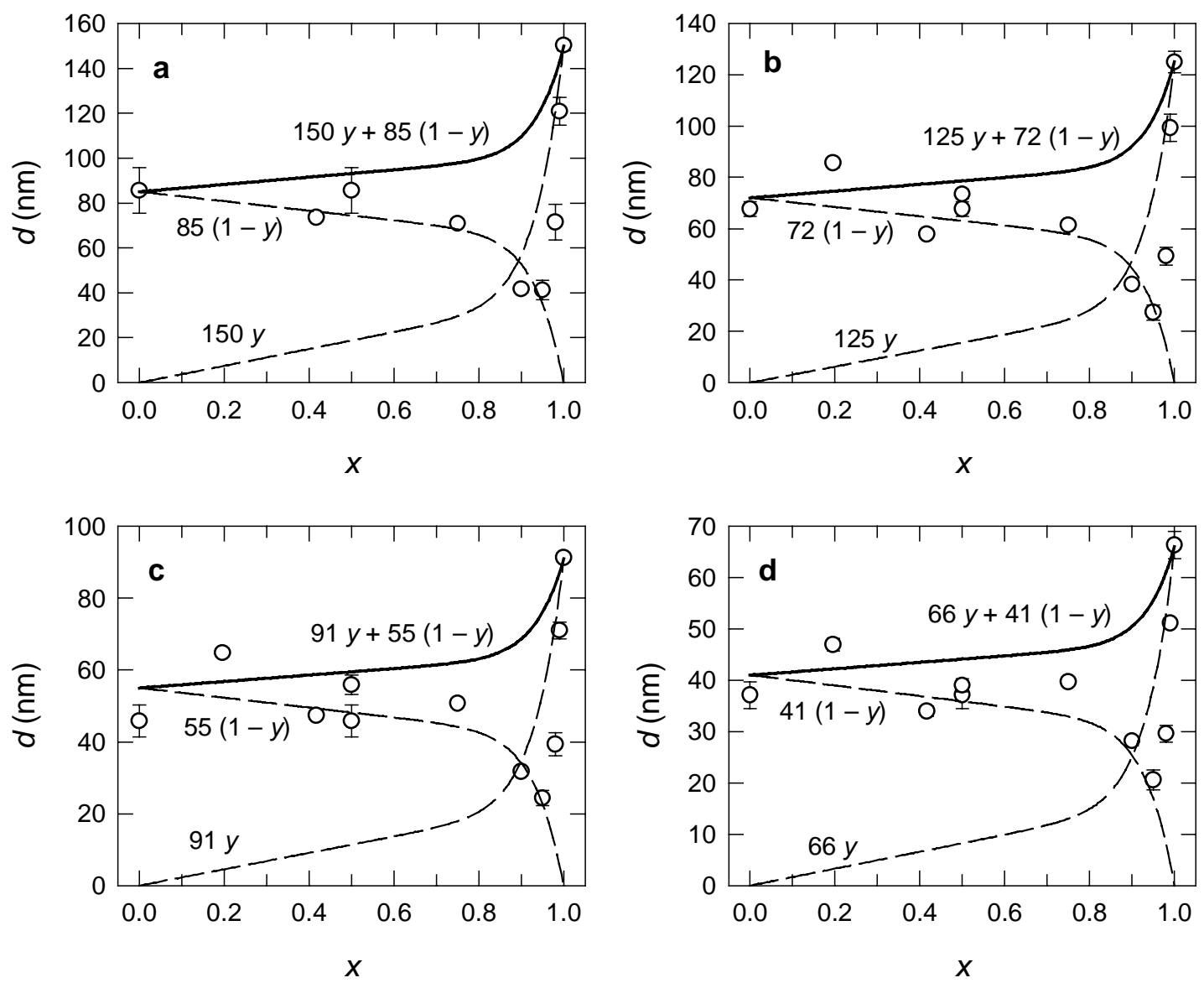

Figure A2. Thickness of PEI-[(HA $\left.\left.\mathrm{HA}_{\mathrm{x}}-\mathrm{PSS}_{1-\mathrm{x}}\right)-\mathrm{PLL}\right]_{\mathrm{n}}$ films as a function of the molar fraction of HA in the blended polyanion solution. The measurements, performed by ellipsometry, are represented by open disks (same as in Figure 2) for $n=14$ (a), 12 (b), 10 (c) and 8 (d). The dashed lines represent either $\alpha y$ or $\beta(1-y)$, as indicated by the labels. The solid line represents $\alpha y+\beta(1-y)$. 
With the hypothesis that the thickness depends on the mass fraction, strongly supported by our observation, we draw also in Figure A2 the quantity $\alpha y$, where $\alpha$ means the thickness when the polyanion solution contains only HA $(x=1)$, which should represent the contribution of HA to the blended film thickness if the two polyelectrolytes contributed independently to it. The values of $\alpha$ are given in Table 1 . In fact, it follows from Figure A2 that HA does practically not contribute to the thickness as long as $x \leq 0.90$, although this polyanion is present in the film (Figure A1), and that its contribution becomes significant only for larger values of $x$, though with an efficiency less than $y$ except when $x=1$. This is further emphasized if we draw $\alpha y+\beta(1-y)$ as a function of $x$, based on the mere additivity of HA and PSS as far as their contribution to the film thickness is concerned (Figure A2). Therefore, the results given in Figure 2 and Figure 5 might appear mutually contradictory.

It follows from the above comment that HA participates to the film thickness at a rate less than its relative abundance, $y$, in the film. Therefore, we still express the film thickness as a combination of $\alpha$ and $\beta$ but, instead of the weight $y$ for $\alpha$, we introduce a weight function aimed at expressing that the expected contribution $\alpha y$ is actually reduced by the presence of PSS in the film. An empirical choice for this function is $y^{\mathrm{q}}$. Thereby, the expression of the thickness writes:

$$
d=\alpha y^{\mathrm{q}}+\beta(1-y),
$$

where $q$ is an adjustable parameter, and $y$ is given by Eq. (A1). The requirement that $d$ goes through a minimum at the value $x_{0}$ of $x$ leads to:

$$
q y_{0}^{\mathrm{q}-1}=\frac{\beta}{\alpha},
$$

where $y_{0}=y\left(x_{0}\right)$. Equation (A3) with $a=0.25, p=15, \alpha$ and $\beta$ given in Table 1 , and $q$ adjusted to 6, leads to the thickness predictions shown in Figure A3 together with the 
measured thicknesses as in Figure A2. As can be seen, the modeled curves follow fairly the trends delineated by the measurements in the four cases $n=14,12,10$ and 8 . Moreover, it is remarkable that the only a priori fully free parameter, $q$, can be kept constant. This suggests that $q$ is more than a fitting parameter, i.e. that the function $y^{\mathrm{q}}$ might have a physical meaning, even though it is far from being understood as to yet. Further experiments with other molar masses of the polyanions could shed light on this point.
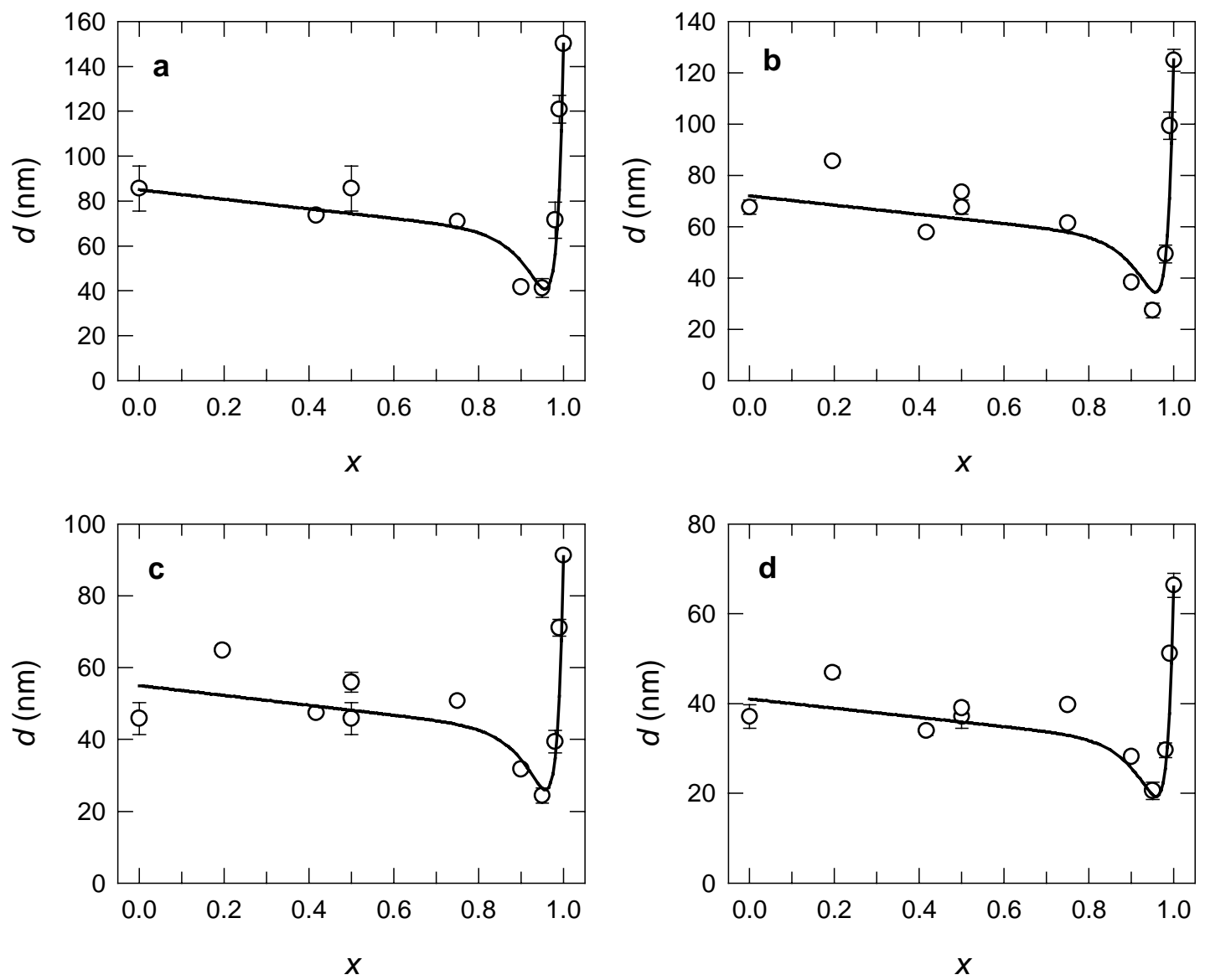

Figure A3. Thickness of PEI-[(HA $\left.\left.\mathrm{HASS}_{1-\mathrm{x}}\right)-\mathrm{PLL}\right]_{\mathrm{n}}$ films as a function of the molar fraction of HA in the blended polyanion solution. The measurements, performed by ellipsometry, are represented by open disks (same as in Figure 2) for $n=14$ (a), 12 (b), 10 (c) and 8 (d). The solid line represents $\alpha y^{\mathrm{q}}+\beta(1-y)$ (Eq. (A3)). 
Table 1. Parameters $\alpha$ and $\beta$ used to reproduce the experimental film thicknesses (Figure A3), together with the only actual free parameter $q$ whose optimal value could be kept fixed ( $q=$ 6.0) in Eq. (A3). Note that because the HA/PLL film is more strongly exponential than the PSS/PLL film (Figure 1), the ratio $\beta / \alpha$ decreases when $n$, the number of pairs of layers, increases. The minimum thickness predicted by Eq. (A3) is obtained for $x=x_{0}$ which is the root of Eq. (A4) to \pm 0.0005 .

\begin{tabular}{ccccc}
\hline$n$ & $\alpha(\mathrm{nm})$ & $\beta(\mathrm{nm})$ & $\beta / \alpha$ & $x_{0}$ \\
\hline 14 & 150 & 85 & 0.567 & 0.956 \\
12 & 125 & 72 & 0.576 & 0.957 \\
10 & 91 & 55 & 0.604 & 0.958 \\
8 & 66 & 41 & 0.621 & 0.958 \\
\hline
\end{tabular}

In summary, the phenomenological model proposed in this section allows reconciling the results given in Figure 2 and Figure 5. The key point in the reasoning is that the thickness of the film is dominated by its mass content in PSS up to $x \approx 0.90$ although HA is present too. More specifically, the thickness decreases with the PSS mass content, but this decrease is not compensated by the increasing amount of HA incorporated in the film until the critical fraction $x_{0} \approx 0.95-0.96$ is reached in the blended polyanion solution, to which corresponds $y \approx$ 0.5. So, not only is PSS preferentially incorporated in the film, but it does, in addition, hinder the participation of the HA to the thickness as long as the mass balance in the film is in favor of PSS. Why the construction proceeds in this way remains, however, an open question. 